This item was submitted to Loughborough's Research Repository by the author.

Items in Figshare are protected by copyright, with all rights reserved, unless otherwise indicated.

\title{
Multiphysics simulation of the effect of leaflet thickness inhomogeneity and material anisotropy on the stress-strain distribution on the aortic valve
}

\section{PLEASE CITE THE PUBLISHED VERSION}

https://doi.org/10.1016/j.jbiomech.2016.02.041

\section{PUBLISHER}

Crown (C) Published by Elsevier BV

VERSION

AM (Accepted Manuscript)

\section{PUBLISHER STATEMENT}

This work is made available according to the conditions of the Creative Commons Attribution-NonCommercialNoDerivatives 4.0 International (CC BY-NC-ND 4.0) licence. Full details of this licence are available at: https://creativecommons.org/licenses/by-nc-nd/4.0/

\section{LICENCE}

CC BY-NC-ND 4.0

\section{REPOSITORY RECORD}

Joda, Akram, Zhongmin Jin, Axel Haverich, Jon Summers, and Sotiris Korossis. 2019. "Multiphysics Simulation of the Effect of Leaflet Thickness Inhomogeneity and Material Anisotropy on the Stress-strain Distribution on the Aortic Valve". figshare. https://hdl.handle.net/2134/37193. 


\section{Multiphysics Simulation of the Effect of Leaflet Thickness \\ Inhomogeneity and Material Anisotropy on the Stress-Strain \\ Distribution on the Aortic Valve}

Akram Joda $^{1,2}$, Zhongmin $\mathrm{Jin}^{1,3}$, Axel Haverich ${ }^{4}$, Jon Summers ${ }^{5}$, Sotirios Korossis K $^{1,4,6^{*}}$

${ }^{1}$ Institute of Medical and Biological Engineering, University of Leeds, LS2 9JT, Leeds, UK

${ }^{2}$ College of Engineering, King Faisal University, Hofuf- Al-Ahassa, 31982, Kingdom of Saudi Arabia

${ }^{3}$ State Key Laboratory for Manufacturing System Engineering Xi An Jiao Tong University Xi An

China

${ }^{4}$ Department of Cardiothoracic, Transplantation and Vascular Surgery Hannover Medical School, Carl-Neuberg-Strasse 1, Hannover, 30625, Germany

${ }^{5}$ Institute of Engineering Thermofluids, Surfaces and Interfaces, University of Leeds, LS2 9JT, Leeds, UK

${ }^{6}$ Lower Saxony Centre for Biomedical Engineering Implant Research and Development, Hannover Medical School, Feodor-Lynen-Strasse 31, Hannover, 30625, Germany

*Corresponding author: Tel: +44 511532 8258, Email: korossis.sotirios@mh-hannover.de

Keywords: aortic valve, fluid-structure interaction, Arbitrary Lagrangian Eulerian, anisotropic material model

Word counts: 3995 


\section{Abstract}

This study developed a realistic 3D FSI computational model of the aortic valve using the fixed-grid method, which was eventually employed to investigate the effect of the leaflet thickness inhomogeneity and leaflet mechanical nonlinearity and anisotropy on the simulation results. The leaflet anisotropy and thickness inhomogeneity were found to significantly affect the valve stressstrain distribution. However, their effect on valve dynamics and fluid flow through the valve were minor. Comparison of the simulation results against in-vivo and in-vitro data indicated good agreement between the computational models and experimental data. The study highlighted the importance of simulating multi-physics phenomena (such as fluid flow and structural deformation), regional leaflet thickness inhomogeneity and anisotropic nonlinear mechanical properties, to accurately predict the stress-strain distribution on the natural aortic valve. 


\section{Introduction}

Over the last four decades, there has been a considerable interest in developing realistic computational models of heart valves, with a view to understanding their function in normal and pathological conditions, aiding the development of artificial valves, and optimizing surgical procedures (Friedman et al. 2010). In spite of recent advances in computational resources and numerical techniques, the development of physiologically-relevant computational models of heart valves still represents a challenge, due to their complex geometries and large deformations during the cardiac cycle.

A number of constitutive equations have been employed in modelling the biomechanics of the native and bioprosthetic aortic valve leaflets, including linear elastic (Gould et al. 1976; Cataloglu et al. 1977; Gnyaneshwar et al. 2002), bilinear or multi-linear elastic (Hamid et al. 1986; Mohammadi et al. 2009), linear orthotropic (Grande et al. 1998; Katayama et al. 2008; Kalyana et al. 2015), nonlinear elastic (Black et al. 1991; Carmody et al. 2006), isotropic matrix with fiberreinforcement (De Hart et al. 2003; Driessen et al. 2007) and Fung-type nonlinear anisotropic (Sun et al, 2003; Kim et al. 2006; Labrosse et al. 2015). Patterson et al. (Patterson et al. 1996) reported that using the nonlinear elastic model had a significant effect on leaflet behavior and stress magnitude compared to the linear elastic model. Burriesci et al. (Burriesci et al. 1999) compared the behavior of a bicuspid pericardial prosthetic heart valve using isotropic nonlinear and orthotropic nonlinear material models and found that orthotropy has a great impact on the stress distribution. A similar finding was reported by Li et al. (Li et al. 2001) on a trileaflet aortic valve.

The thickness of the aortic valve leaflet varies regionally (Cataloglu et al. 1977; Thubrikar 1990; Grande et al. 1998; Wang et al. 2008). A number of numerical studies compared the stress distribution on the aortic valve using uniform and non-uniform leaflet thickness and found significant differences in the stress distribution and magnitude (Cataloglu et al. 1977; Li et al. 2001; Luo et al. 2003). However, these studies were static and only the maximum transvalvular pressure gradient was considered. In addition, these studies did not account for the leaflet coaptation, or most vitally, the interaction of the fluid with the valve structure. 
A numerical simulation that closely represents the physiological aortic valve dynamics, incorporating leaflets opening and closing and root dilation, requires coupling the governing equations of the valve structure deformation and blood flow using a fluid-structure-interaction (FSI) methodology. Over years, many FSI methods have been developed and there are mainly categorized into two general approaches; the Arbitrary Lagrangian Eulerian (ALE) and fixed-mesh (Eulerian) methods. In The ALE approach, two conforming meshes are used for the fluid and the structure while in the Eulerian two separate meshes are used. The ALE method has been successfully applied to only bileaflet mechanical heart valves where the leaflets were modelled as rigid bodies (Dumont et al. 2007; Nobili et al. 2008; Guivier-Curien et al. 2009). The main drawback of this method is its inability to cope with large deformation problems. Owing to this, this method failed in the FSI modeling of natural and prosthetic valves, where the leaflets undergo large displacement during the systolic phase and large deformation during the diastolic phase. Fixedgrid methods, in which the fluid mesh remains unchanged, have been shown to be capable of solving FSI problems where the structure undergoes large deformations in complex geometries. Different fixed-mesh methods have been proposed to study FSI of the aortic valve. These include the immersed boundary method (IBM) (Peskin 1972; Griffith et al. 2009), sharp-interface immersed boundary method (Borazjani 2013), sharp-interface level set method (Vigmostad et al. 2010), fictitious domain (De Hart et al. 2003; Loon 2004) and Multi-Material Arbitrary Lagrangian Eulerian (MM-ALE) methods (Nicosia et al. 2003; Carmody et al. 2006; Ranga et al. 2006; Weinberg and Mofrad 2007). Previous fixed-grid studies have either used uniform leaflet thickness, nonphysiological boundary conditions, or simplified leaflet material models. In addition, they have not been validated in detail, apart from the case of the study by Weinberg and Kaazempur Mofrad (Weinberg \& Kaazempur Mofrad 2007).

In the present work, 3D FSI models of the natural aortic valve were developed using the fixed-grid FSI method (MM-ALE) to study the effect of the non-uniform leaflet thickness and leaflet mechanical anisotropy properties on the leaflet stress-strain distribution, valve dynamics and fluid flow through the valve. 


\section{Material and Methods}

\section{FSI Method}

The explicit finite element software LS-DYNA (971, LSTC, Livermore, CA) that employs the fixed-mesh MM-ALE method was utilized in the simulations. This method used a Split-Operator method to split the computation in each time step into two steps. Initially, the conservation equations were solved in the Lagrangian formulation by setting the mesh velocity to the fluid velocity, and the changes in the internal energy and velocity that occurred due to the external and internal forces were computed. Consequently, the equilibrium equations of the momentum and energy were (Souli et al. 2000; Aquelet et al. 2006):

$$
\begin{aligned}
& \frac{\partial \rho v_{i}}{\partial t}=-\frac{\partial \sigma_{i j}^{f}}{\partial x_{j}}+F_{i} \\
& \frac{\partial e}{\partial t}=\sigma_{i j}^{f} \frac{\partial v_{i}}{\partial x_{j}}+F_{i} v_{i}
\end{aligned}
$$

where $\sigma_{i j}^{f}=-p \delta_{i j}+\mu\left(\frac{\partial v_{i}}{\partial x_{j}}+\frac{\partial v_{j}}{\partial x_{i}}\right)$ and $e$ was the internal energy. The second step was the advection step, in which all nodes were remapped back to their initial positions and the transport of internal energy, mass and momentum through elements boundaries were computed. The transport equations of a variable $\phi$ for the advection step were:

$$
\begin{gathered}
\frac{\partial \phi}{\partial t}+\left(v_{i}-v_{i}^{g}\right) \frac{\partial \phi}{\partial x_{i}}=0 \\
\phi\left(x_{i}, 0\right)=\phi_{0}(x)
\end{gathered}
$$

Two conditions have to be applied to the interface, the kinematic (that the displacement of the fluid is equal to the displacement of the structure) and dynamic conditions (the normal fluid and solid stresses are equal on the interface).

The FSI the coupling is attained using the penalty method which is based on energy conservation and force equilibrium at the interface (Aquelet et al. 2006). The coupling problem is solved as a contact problem, for that at each time step the forces of the fluid on the structure and the forces of the structure on the fluid are computed at the interface and added to the nodal forces. 
At the interface, a resisting force is applied to the slave node relative to its penetration through the master. The same amount of this force is applied to the nodes of the master elements, but in the opposite direction.

\section{Geometry and mesh}

The 3D geometry of the aortic valve was created using the geometrical parameters reported in (Thubrikar 1990; Swanson and Clark 1974). An idealized aortic valve geometry was used in this study by assuming that it comprised three identical sinuses and leaflets ( $120^{\circ}$ symmetry) and each sinus-leaflet portion had a symmetry plane along its central line (Figure 1a). Therefore, only onesixth of the aortic valve geometry was considered in the numerical simulations as in previous studies (Weinberg \& Kaazempur Mofrad 2007; De Hart et al. 2003; Loon 2009). For the leaflet thickness, two configurations were adopted. In the first, the leaflet thickness was assumed to be uniform with a value of $0.5 \mathrm{~mm}$, whereas, in the second, the leaflet thickness was varied within the physiological range obtained in-vitro (Figure 1 b) (Cataloglu et al. 1977; Thubrikar 1990; Grande et al. 1998; Wang et al. 2008). Figure 1 c \& d show the values as measured in Grande et al. (Grande et al 2008) and Wang el al.(Wang et al. 2008).

Two separate meshes representing the fluid and valve domain were used. A sensitivity analysis was conducted to determine the effect of fluid and solid meshes refinement on the valve results. Table 1 shows the three models used in the study and the number of fluid and solid elements. The analysis indicated that Mod-2 with one element across the leaflet thickness and two elements across the aortic wall thickness can accurately predict the valve dynamics, fluid flow, and stress-strain distribution within a reasonable computational time (Figure $2 \mathrm{a}-\mathrm{d}$ ). Owing to this, all simulations were conducted using Mod-2. The structural parts (leaflet and aortic root) were meshed using 3036 8-node brick elements and the fluid parts were meshed using 12332 8-node brick elements.

\section{Boundary conditions}

Symmetry boundary conditions were applied to the fluid and valve nodes on the two symmetry planes (Figure 3a). The aortic root was fixed in translation and rotation in all directions 
from its top and bottom ends. Under the incompressibility assumption, the blood flow was only driven by the pressure gradient between the aorta and the left ventricle, using the physiological transvalvular pressure gradient applied at the inlet (Levick 2010) (Figure 3b), whereas a zero pressure was applied at the outlet.

\section{Fluid Properties}

The fluid was modeled as laminar Newtonian and marginally compressible, with a density and viscosity within the physiological range; $1000 \mathrm{~kg} / \mathrm{m}^{3}$ and 0.004 Pa.s (Narracott et al. 2010), respectively. The speed of sound was reduced from its physiological values of $1500 \mathrm{~m} / \mathrm{s}$ to $20 \mathrm{~m} / \mathrm{s}$. However, the incompressibility of the flow was maintained by keeping the Mach number below 0.1 . The reduction of the speed of sound was found to have a negligible effect on the FSI results, however, the computation time was reduced by a factor of three (Joda 2011).

\section{Materials Models}

The aortic root was modeled as an isotropic hyperelastic material using the Mooney-Rivlin model with $\mathrm{C}_{1}=0.1 \mathrm{MPa}$ (Weinberg \& Kaazempur Mofrad 2007). In the case of the leaflet, three material models, available in the LS-DYNA material library, were used as described below.

\section{Nonlinear isotropic (*MAT_OGDEN_RUBBER)}

Using this model, the leaflet was assumed to be nonlinear isotropic and the first order Ogden model was used to fit the circumferential stress-strain curve of the aortic valve leaflet obtained from tensile tests (Korossis et al. 2002):

$$
W=\frac{\mu}{\alpha}\left(\lambda_{1}^{\alpha}+\lambda_{2}^{\alpha}+\lambda_{3}^{\alpha}-3\right)+\frac{1}{2} K(J-1)^{2}
$$

where $\lambda_{1}, \lambda_{2}$, and $\lambda_{3}$, were the principal stretches in the three directions, $\mu_{p}$ and $\alpha_{p}$ the model constants, $K$ the bulk modulus and $J=\lambda_{1} \lambda_{2} \lambda_{3} \approx 1$. A special function was written in MATLAB (MathWorks Inc) using the trust-region-reflective algorithm to characterize the Ogden model parameters by minimizing the sum of the error squared. A good fit was found with the constants $\mu_{1}$ $=59782.4 \mathrm{~Pa}$ and $\alpha_{1}=12.97$ (Figure 4a). 


\section{Fiber-reinforced model ('MAT_SOFT_TISSUE)}

In this model, the leaflet was modeled as an isotropic matrix reinforced by a collagen fiber family (Weiss et al. 1996; Puso \& Weiss 1998). The isotropic part of this model was modeled using the Mooney-Rivlin material model and an exponential function was used to account for the behavior of crimped collagen fibers:

$$
W=C_{1}\left(I_{1}-3\right)+C_{2}\left(I_{2}-3\right)+F\left(\lambda_{f}\right)+\frac{1}{2} K[\ln (J)]^{2}
$$

where $\lambda_{f}$ was the stretch along the fiber direction and $F\left(\lambda_{f}\right)$ described the fiber behavior using the following equation:

$$
F\left(\lambda_{f}\right)=\left\{\begin{array}{cc}
0 & \lambda_{f}<1 \\
\frac{C_{3}}{\lambda_{f}}\left[\exp \left(C_{4}\left(\lambda_{f}-1\right)\right)-1\right] & 1 \leq \lambda_{f}<\lambda_{f}^{*} \\
\frac{1}{\lambda_{f}}\left(C_{5} \lambda_{f}+C_{6}\right) & \lambda_{f} \geq \lambda_{f}^{*}
\end{array}\right\}
$$

where $C_{3}, C_{4}, C_{5}$ and $C_{6}$ were constants and $\lambda_{f}^{*}$ was the critical stretch value at which the fibers were straightened. It was assumed that the fibers were unable to sustain compression and so the fiber term was only active when the stretch was greater than one $\left(\lambda_{f}>1\right)$. It was assumed that the collagen fibers in the leaflet were aligned along the circumferential direction (Figure 2a). A MATLAB function was written to fit the model to experimental circumferential stress-strain data (Korossis et al. 2002) using $\mathrm{C}_{1}=0.06 \mathrm{MPa}, \mathrm{C}_{2}=0.0, \mathrm{C}_{3}=1.17431 \mathrm{e} 5 \mathrm{~Pa}$ and $\mathrm{C}_{4}=9.19$ (Figure $4 \mathrm{a}$ $\& b)$.

\section{Nonlinear Anisotropic Fung-Type model (MAT_HEART_TISSUE)}

This Fung-type constitutive model was transversely isotropic and hyperelastic (Fung et al. 1979; Guccione et al. 1991) with a strain energy function defined as a function of Green-Lagrange strain components:

$$
W=\frac{C_{h}}{2}\left(e^{Q}-1\right)+\frac{1}{2} K(J-1)^{2}
$$

where $Q=b_{1} E_{11}^{2}+b_{2}\left(E_{22}^{2}+E_{33}^{2}+E_{23}^{2}+E_{32}^{2}\right)+b_{3}\left(E_{12}^{2}+E_{21}^{2}+E_{13}^{2}+E_{31}^{2}\right)$, and $C_{h}, b_{1}, b_{2}$, and $b_{3}$ were the model parameters. This model was used to model the mechanical anisotropy of the leaflet. The 
model was used to fit the circumferential and radial stress-strain data of the aortic valve leaflet obtained from tensile tests (Korossis et al. 2002). The parameters of the model were identified as described above, and a good fit was found with $C_{h}=5.89 \mathrm{e} 2 \mathrm{kPa}, b_{1}=4.19, b_{2}=0.299$ (Figure 4 a \&b). Since $b_{3}$ could not be captured by the tensile test, it was assumed to be equal to $b_{2}$ (Labrosse et al. 2010).

\section{Results}

The mean computed stress at the base, commissure and belly at the peak diastolic pressure for the uniform leaflet thickness and physiological leaflet thickness (non-uniform) aortic valve models is shown in Figure 5a. The comparison was conducted using the maximum principal stress and the effective strain. The valve model with the physiological leaflet thickness predicted $33 \%$ lower stress at the commissure and a $100 \%$ higher stress at the belly compared to the uniform thickness model. Similarly, the valve model with the non-uniform leaflet thickness predicted $27 \%$ lower strain at the commissure and $80 \%$ higher strain at the belly compared to the uniform thickness model (Figure 5b). Moreover, investigation of the effect of the three different leaflet material models on the stress-strain distribution on the leaflet at peak systole and peak diastole is presented in Figure. 6. All three models predicted high stress magnitudes at the lower end of the leaflet commissures. The stress magnitude in the isotropic model was approximately $14 \%$ and $25 \%$ higher compared to the fiber-reinforced model and the Fung-type model, respectively. In addition, it was observed that the fiber-reinforced model and the Fung-type model predicted comparable stress distributions. In contrast, the isotropic model predicted lower strain compared to the other two models at peak systole. The maximum strain value of the isotropic model was about $50 \%$ lower than the anisotropic models.

Following valve closure, at the beginning of diastole, the leaflets started to coapt and slide downward when the pressure continued to drop in the left ventricle. During coaptation, the leaflet stress and strain increased with the drop of transvalvular pressure. At peak diastole, the fiberreinforced and the Fung-type models demonstrated similar stress distributions with high stress concentration at the leaflet belly region (Figure 6). However, the isotropic model predicted another high stress region at the commissure. The highest stress in the isotropic model was found to be 
$12 \%$ and $15 \%$ higher than the highest stress in the fiber-reinforced and Fung-type models, respectively. In the Fung-type model, the mean stress magnitude at the belly was found to be about $89 \%$ and $71 \%$ higher than the mean stress values at the base and the commissural regions. In contrast to the stress, the strain at peak diastole was found to be higher in the fiber-reinforced model and the Fung-type model when compared to the isotropic model. However, in all three material models the location of the higher strain was predicted to occur at the belly, and the lower strain at the free edge. The strain magnitude at the belly region for the isotropic model was found to be about $32 \%$ and $25 \%$ lower than the fiber-reinforced and the Fung-type models, respectively.

The effects of the leaflet material model on the stress and strain distribution on the aortic root at peak systole and peak diastole are illustrated in Figure 7. During systole, the aortic root dilated and moved outward, mainly the commissures, when the pressure rose in the ventricle. For all the three material models, the stress was higher at the lower part of the aortic root (leaflet attachment to the aortic wall region) and at the inter-leaflet triangles, and lower in the sinuses. At peak systole, the location of the highest stress and strain magnitudes was consistent in the three models and located at the bottom of the commissures. At this instant, the maximum stress magnitude of the nonlinear isotropic model was about $25 \%$ and $34 \%$ higher compared to the fiber-reinforced and the Fung-type models, respectively. Consequently, the nonlinear isotropic model predicted $28 \%$ and $35 \%$ higher strain magnitudes than the fiber-reinforced and Fung-type models, respectively. During diastole, the aortic root moved inward, especially at the commissures, due to the pulling force of the leaflet. Therefore, high stress magnitudes were found in the leaflet attachment region and the commissures.

The fluid velocity vectors during systole for the three models are illustrated in Figure 8. During the fluid acceleration phase, the commissures moved outward and the valve opened rapidly. When the leaflet reached its maximum radial position, a recirculation zone was formed at the top of the center of the leaflet free edge, moving downstream with the continued flow acceleration. After the velocity reached its peak value, the flow started to decelerate rapidly but at a slower rate than the initial acceleration phase. During the closing phase, some differences were found in the leaflet dynamics between the three models. The fiber-reinforced model started the closing phase earlier than the other two models. Another recirculation zone was observed in the ascending aorta and 
moved upstream to the sinus during the valve closure, which may aid valve closure as reported by (Bellhouse \& Bellhouse 1968; Bellhouse \& Talbot 1969).

The opening/closing characteristics of the three leaflet material models were compared to invivo echocardiographic data (De Paulis et al. 2001) (Figure 9a), in terms of the rapid valve opening time (RVOT), rapid valve closing time (RVCT), ejection time (ET), rapid valve opening velocity (RVOV) and rapid valve closing velocity (RVCV). All models were able to predict the opening and closing characteristics of the aortic valve with a reasonable accuracy, with similar RVOT and RVOV. However, the fiber-reinforced model demonstrated a slower closing phase than the other two models. All models closed at lower diastolic transvalvular pressures compared to the in-vivo measurements, as evidenced by the shorter ETs.

The aortic root at the commissures was found to move outward during systole and inward during diastole. The percentage difference in the commissural diameter between peak systole and peak diastole was measured experimentally in-vivo to be $5 \pm 1.3 \%$ (De Paulis et al. 2001). The corresponding values in the isotropic, fiber-reinforced and Fung-type models were $6.3 \%, 6.2 \%$ and $5.9 \%$ respectively, which are within the statistical error of, the in-vivo data. The circumferential and radial stretches of the leaflet belly during the cardiac cycle for the three material models are demonstrated in Figure $9 b$ and 9c, respectively, together with the experimental data from an invitro hydrodynamic study with porcine aortic valves (Kim 2009). In these experiments, the reference valve configuration was taken as the unloaded state of the valve under zero pressure. Thus, all experimental and FSI stretches were normalized by the end-diastolic stretch values. For the FSI models, the circumferential and radial stretches of the 16 elements in the belly region were averaged. The error bars in the experimental and FSI model curves represent standard deviations. All models showed very similar circumferential stretch behavior during the cardiac cycle and were in good agreement with the in-vitro experiments. However, differences were observed in the radial direction between the FSI models, with the isotropic model predicting lower stretches. This was expected due to the stiffer model used (isotropic Ogden model) to fit the experimentally-obtained circumferential stress-strain curve. However, the fiber-reinforced and Fung-type models predicted similar stretches to the experimental values. 
The averaged fluid velocity at the inlet of the aorta was also compared to in-vivo aortic velocity measurements (Levick 2010) (Figure 9d). During the initial systolic phase, rapid acceleration of the fluid through the valve was observed in both the experimental and FSI models. The peak systolic velocity of the isotropic model, fiber-reinforced model and Fung-type model were higher by $3.1 \%, 8.3 \%$ and $8.2 \%$, respectively, than the in-vivo measurements. All models predicted shorter forward flow times, which agreed with the results of shorter ejection times (Figure 9a). However, all material models showed good agreement with the in-vivo data during the acceleration phase and similar trends during the deceleration phase.

\section{Discussion}

The MM-ALE was employed to study FSI of the natural aortic valve with a view to investigating the influence of a non-uniform leaflet thickness and the anisotropy of the leaflet mechanical properties on the stress-strain distribution, valve dynamics and blood flow.

In the natural aortic valve, the leaflet thickness is known to vary regionally. The computational simulations indicated that the adoption of a physiological thickness variation across the leaflet significantly affected the stress-strain distribution on the aortic valve leaflet, especially at the commissure and the belly regions. In addition, the higher stresses and strains were predicted for the thinner leaflet regions. Similar findings were reported in previous static structural numerical studies (Cataloglu et al. 1977; Li et al. 2001; Luo et al. 2003). The present work also demonstrated that the models utilizing the average leaflet thickness can produce inaccurate stress-strain values. The non-uniform thickness model predicted about $90 \%$ and $80 \%$ higher stress and strain values compared to the uniform thickness model at the peak diastolic transvalvular pressure. However, no considerable differences were found for the aortic valve dynamics and fluid patterns across the valve.

A number of structural studies have showed that material anisotropy has a great effect on the stress-strain distributions and magnitudes (Burriesci et al. 1999; Li et al. 2001; Kim et al. 2006). In the present study, the two anisotropic leaflet models showed very similar stress-strain distributions, whereas the isotropic model was stiffer (higher stress for lower strain). During peak diastole, all three material models predicted higher stress values in the belly region (where the leaflet is 
thinner). However, additional high stress regions were found with the isotropic model at the commissures, which was also reported previously (Li et al. 2001). The results from the three material models were compared to in-vivo and in-vitro data. No significant differences were found in the opening and closing times and velocities between the three models and all were in good agreement with the in-vivo data. The study found the that although the recirculation might aid valve closure, however, it is the adverse pressure gradient during diastole is the main mechanism as reported by (Van Steenhoven \& Van Dongen 1979; Lee \& Talbot 1979).

Although the models developed in this study produced realistic and accurate multi-physics simulations of the aortic valve, a number of assumptions were employed for simplicity. The geometrical model of the valve was created using idealized parameters. The natural aortic valve is known to be complex and varying in dimensions and size between individuals. In addition, a $120^{\circ}$ valve symmetry was assumed in order to reduce the computational time further. Morphological differences among the three sinuses and leaflets have been reported in the literature which, consequently, resulted in variations of the stress-strain distributions (Grande et al. 1998; Conti et al. 2010). However, these differences were assumed to be small compared to the large deformation and high stress that occurred in the valve. In addition, these anatomical differences have not yet been quantified biomechanically. Moreover, the mechanical properties of the aortic wall and leaflet have been shown to be nonlinear and anisotropic and vary from region to region. To the best of the authors' knowledge, the regional mechanical properties of the whole aortic valve have yet to be fully quantified. The leaflet is composed of three layers across its thickness, including the fibrosa, spongiosa and ventricularis, with each of the layers exhibiting distinct mechanical properties (Stella and Sacks 2007). Taking into account the variation in the mechanical properties across the leaflet thickness would enhance the accuracy of the computed stress and strain distributions. However, in this work the mechanical properties were assumed to be isotropic across the leaflet thickness. Moreover, the aortic root was assumed to be nonlinear and homogenous and the same properties were used for the whole root. In terms of the fluid model, it was assumed laminar, mildly compressible and Newtonian. However, the flow in the natural aortic valve can become turbulent or transitional, especially at peak systole. Laminar and 
compressible assumptions were used in this work since the FSI solver was only available for compressible and laminar flows.

\section{Conclusions}

A realistic computational FSI model of the aortic valve was developed using a fixed-grid method (MM-ALE), and by considering the physiological leaflet thickness, and mechanical nonlinearity and anisotropy. The study highlighted the importance of a multi-physics simulation approach and the necessity to employ the physiological regional leaflet thickness inhomogeneity and anisotropic nonlinear mechanical properties to accurately predict the stress-strain distributions on the aortic valve.

\section{Conflict of interest}

The authors have no conflict of interest.

\section{Acknowledgements}

This work was supported by a Marie Curie EST Fellowship and the Leeds Centre of Excellence in Medical Engineering, funded by the Wellcome Trust and the Engineering and Physical Sciences Research Council, WT088908/z/09/z. 


\section{References}

Aquelet, N., Souli, M. \& Olovsson, L., 2006. Euler-Lagrange coupling with damping effects: Application to slamming problems. Computer Methods in Applied Mechanics and Engineering, 195(1-3), pp.110-132.

Bellhouse, B.J. \& Bellhouse, F.H., 1968. Mechanism of Closure of the Aortic Valve. Nature, 217(5123), pp.86-87.

Bellhouse, B.J. \& Talbot, L., 1969. The fluid mechanics of the aortic valve. Journal of Fluid Mechanics, 35(04), pp.721-735.

Burriesci, G., IC, H. \& EA, P., 1999. Influence of anisotropy on the mechanical behaviour of bioprosthetic heart valves. J Med Eng Technol, 23(6), pp.203-215.

Cataloglu, A., Clark, R.E. \& Gould, P.L., 1977. Stress analysis of aortic valve leaflets with smoothed geometrical data. Journal of Biomechanics, 10(3), pp.153-158.

Conti, C. a et al., 2010. Dynamic finite element analysis of the aortic root from MRI-derived parameters. Medical engineering \& physics, 32(2), pp.212-21.

Dumont, K. et al., 2007. Comparison of the hemodynamic and thrombogenic performance of two bileaflet mechanical heart valves using a CFD/FSI model. Journal of biomechanical engineering, 129(4), pp.558-65.

Friedman, M.H., Krams, R. \& Chandran, K.B., 2010. Flow Interactions with Cells and Tissues: Cardiovascular Flows and Fluid-Structure Interactions. Annals of Biomedical Engineering, 38(3), pp.1178-1187.

Fung, Y.C., Fronek, K. \& Patitucci, P., 1979. Pseudoelasticity of arteries and the choice of its mathematical expression. American Journal of Physiology - Heart and Circulatory Physiology 
, 237 (5 ), pp.H620-H631.

Grande, K.J. et al., 1998. Stress variations in the human aortic root and valve: the role of anatomic asymmetry. Annals of biomedical engineering, 26(4), pp.534-45.

Griffith, B.E. et al., 2009. Simulating the Fluid Dynamics of Natural and Prosthetic Heart Valves Using the Immersed Boundary Method. International Journal of Applied Mechanics, 01(01), pp.137-177.

Guccione, J.M., McCulloch, A.D. \& Waldman, L.K., 1991. Passive material properties of intact ventricular myocardium determined from a cylindrical model. Journal of biomechanical engineering, 113(1), pp.42-55.

Guivier-Curien, C., Deplano, V. \& Bertrand, E., 2009. Validation of a numerical 3-D fluid-structure interaction model for a prosthetic valve based on experimental PIV measurements. Medical engineering \& physics, 31(8), pp.986-93.

De Hart, J. et al., 2003. A three-dimensional computational analysis of fluid-structure interaction in the aortic valve. Journal of biomechanics, 36(1), pp.103-12.

Joda A. A. O., 2011, "Fluid-Structure Interaction of the Aortic Valve for Tissue Engineering Applications," PhD Thesis, University of Leeds, Leeds.

Kim, H. et al., 2006. Dynamic simulation pericardial bioprosthetic heart valve function. Journal of biomechanical engineering, 128(5), pp.717-24.

Kim, H.S., 2009. Nonlinear Multi-Scale Anisotropic Material and Structural Models for Prosthetic and Native Aortic Heart Valves. Georgia Institute of Technology, PhD Thesis.

Korossis, S.A. et al., 2002. Tissue engineering of cardiac valve prostheses II: biomechanical characterization of decellularized porcine aortic heart valves. The Journal of heart valve disease, 11(4), pp.463-471.

Labrosse, M.R., Lobo, K. \& Beller, C.J., 2010. Structural analysis of the natural aortic valve in dynamics: from unpressurized to physiologically loaded. Journal of biomechanics, 43(10), pp.1916-22.

Labrosse M. R., Beller C.J., Boodhwani M., Hudson C., Sohmer B., Lobo K., 2015, "Subject-415 specific finite-element modeling of normal aortic valve biomechanics from 3D+t TEE images.," 416 Medical Image Analysis 20 (2015),pp 162-172 
Lee, C.S.F. \& Talbot, L., 1979. A fluid-mechanical study of the closure of heart valves. Journal of Fluid Mechanics, 91(01), pp.41-63.

Levick, J.R., 2010. An Introduction to Cardiovascular Physiology, Hodder Arnold.

Li, J., Luo, X.Y. \& Kuang, Z.B., 2001. A nonlinear anisotropic model for porcine aortic heart valves. Journal of biomechanics, 34(10), pp.1279-89.

Loon, R. Van, 2009. Towards computational modelling of aortic stenosis.

Luo, X.Y., Li, W.G. \& Li, J., 2003. Geometrical Stress-Reducing Factors in the Anisotropic Porcine Heart Valves. Journal of Biomechanical Engineering, 125(5), p.735.

Narracott, A.J. et al., 2010. Analysis of a mechanical heart valve prosthesis and a native venous valve: Two distinct applications of FSI to biomedical applications. International Journal for Numerical Methods in Biomedical Engineering, 26(3-4), pp.421-434.

Nobili, M. et al., 2008. Numerical simulation of the dynamics of a bileaflet prosthetic heart valve using a fluid-structure interaction approach. Journal of biomechanics, 41(11), pp.2539-50.

De Paulis, R. et al., 2001. Opening and closing characteristics of the aortic valve after valvesparing procedures using a new aortic root conduit. The Annals of Thoracic Surgery, 72(2), pp.487-494.

Peskin, C.S., 1972. Flow Patterns Around Heart Valves: A Digital Computer Method for Solving the Equations of Motion. Albert Einstein College of Medicine, PhD Thesis.

Puso, M.A. \& Weiss, J.A., 1998. Finite element implementation of anisotropic quasi-linear viscoelasticity using a discrete spectrum approximation. Journal of biomechanical engineering, 120(1), pp.62-70.

Souli, M., Ouahsine, A. \& Lewin, L., 2000. ALE formulation for fluid-structure interaction problems. , 190.

Van Steenhoven, A.A. \& Van Dongen, M.E.H., 1979. Model studies of the closing behaviour of the aortic valve. Journal of Fluid Mechanics, 90(01), pp.21-32.

SWANSON, W.M. \& CLARK, R.E., 1974. Dimensions and Geometric Relationships of the Human Aortic Value as a Function of Pressure. Circulation Research , 35 (6 ), pp.871-882.

Thubrikar, M., 1990. The Aortic Valve, CRC Press.

Wang, L. et al., 2008. Computational simulation of oxygen diffusion in aortic valve leaflet for tissue 
Effect of Leaflet Thickness and Material Anisotropy on the Stress-Strain Distribution on the Aortic Valve

engineering applications. The Journal of heart valve disease, 17(6), pp.700-709.

Weinberg, E.J. \& Kaazempur Mofrad, M.R., 2007. Transient, three-dimensional, multiscale simulations of the human aortic valve. Cardiovascular engineering (Dordrecht, Netherlands), 7(4), pp.140-55.

Weiss, J.A., Maker, B.N. \& Govindjee, S., 1996. Finite element implementation of incompressible, transversely isotropic hyperelasticity. Computer Methods in Applied Mechanics and Engineering, 135(1-2), pp.107-128. 


\section{Figure Captions}

Figure 1. (a) Schematic of the aortic valve showing the geometry and dimensions, (b) computational mesh of the aortic valve leaflet used for uniform and varied thickness models. (c) and (d) Measurements of the leaflet thickness by Grande et al. (Grande et al. 1998) and Wang et al. (Wang et al. 2008) were adopted in this study.

Figure 2. Results of the mesh sensitivity study. (a) Radial displacement of the leaflet tip; (b) axial displacement of the leaflet tip; (c) the maximum flow rate at the outlet; (d) the average values of stress on the leaflet at the base, belly and commissure parts. The error bars indicate standard deviations.

Figure 3. (a) The computational mesh and boundary conditions of the natural aortic valve model showing the fluid domain with the inlet, outlet and aortic root parts (b) Aortic, ventricular and transvalvular pressures (Levick, 2010). The transvalvular pressure gradient was used as the input boundary condition in the FSI models.

Figure 4. Experimental stress-strain relationship of the aortic valve leaflet in the circumferential and radial directions obtained from (Korossis et al.) (a) The nonlinear isotropic (*MAT_OGDEN_RUBBER), fiber-reinforced (*MAT_SOFT_TISSUE) and the Fung-type (*MAT_HEART_TISSUE) material models were fitted the experimental circumferential stress-strain curve, (b) The fiber-reinforced and the Fung-type material models were fitted the experimental radial stress-strain curve.

Figure 5. Comparison of the principal stress (a) and the effective strain (b) at the base, belly and commissure for the models with uniform leaflet thickness and non-uniform leaflet thickness. The error bars indicate the standard deviation of the mean. The average values and standard deviations were computed using 12 elements at the base, 10 elements at the commissure and 16 elements at the belly.

Figure 6. Maximum principal stress and effective strain distributions on the leaflet at (i) peak systole (fully open position) and (ii) peak diastole (fully closed position); a) nonlinear isotropic model, b) fiber-reinforced model, and c) Fung-type model. 
Figure 7. Maximum principal stress and effective strain distributions on the aortic root wall at (i) peak systole and (ii) peak diastole (fully closed position); a) nonlinear isotropic model, b) fiberreinforced model, and c) Fung-type model.

Figure 8. Velocity vectors of fluid flow through the aortic valve during opening and closing phases for (a) nonlinear isotropic model, (b) fiber-reinforced model and (c) Fung-type.

Figure 9. (a) Comparison of the opening and closing time and velocity of the three leaflet material models used with in-vivo experiments. RVOT: rapid valve opening time, RVCT: rapid valve closing time, ET: ejection time, RVOV: rapid valve opening velocity, RVCV: rapid valve closing velocity. (b) Comparison of the circumferential stretch of the leaflet belly of the three leaflet material models to in-vitro experiments (Kim, 2009). The error bars represent the standard deviation. (c) Comparison of the radial stretch of the leaflet belly of the three leaflet material models to in-vitro experiments. The error bars represent the standard deviation. (d) Comparison of average velocity at the aorta for the three models to in-vivo data (Levick, 2010). 

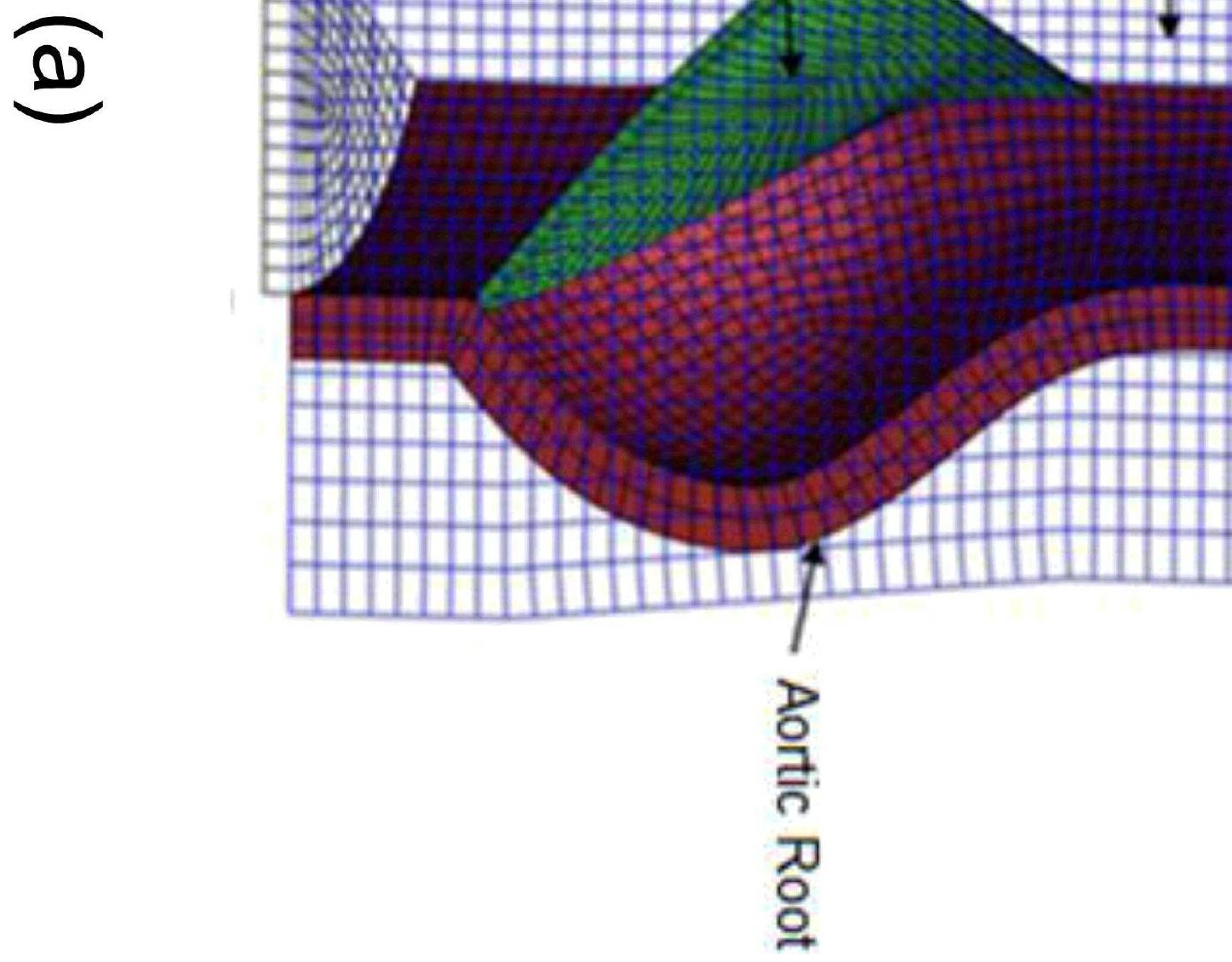

\section{Pressure (mm Hg)}

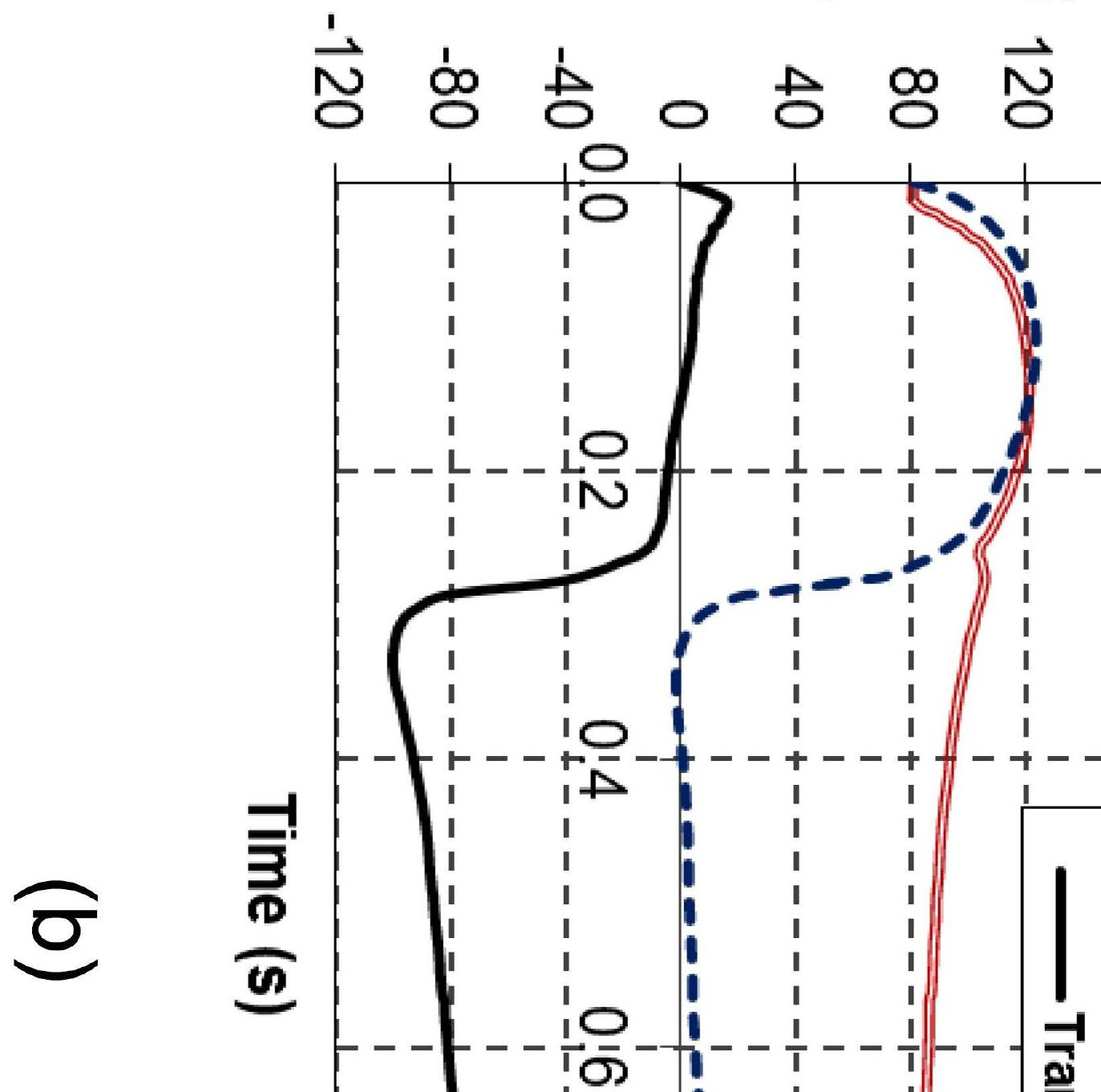



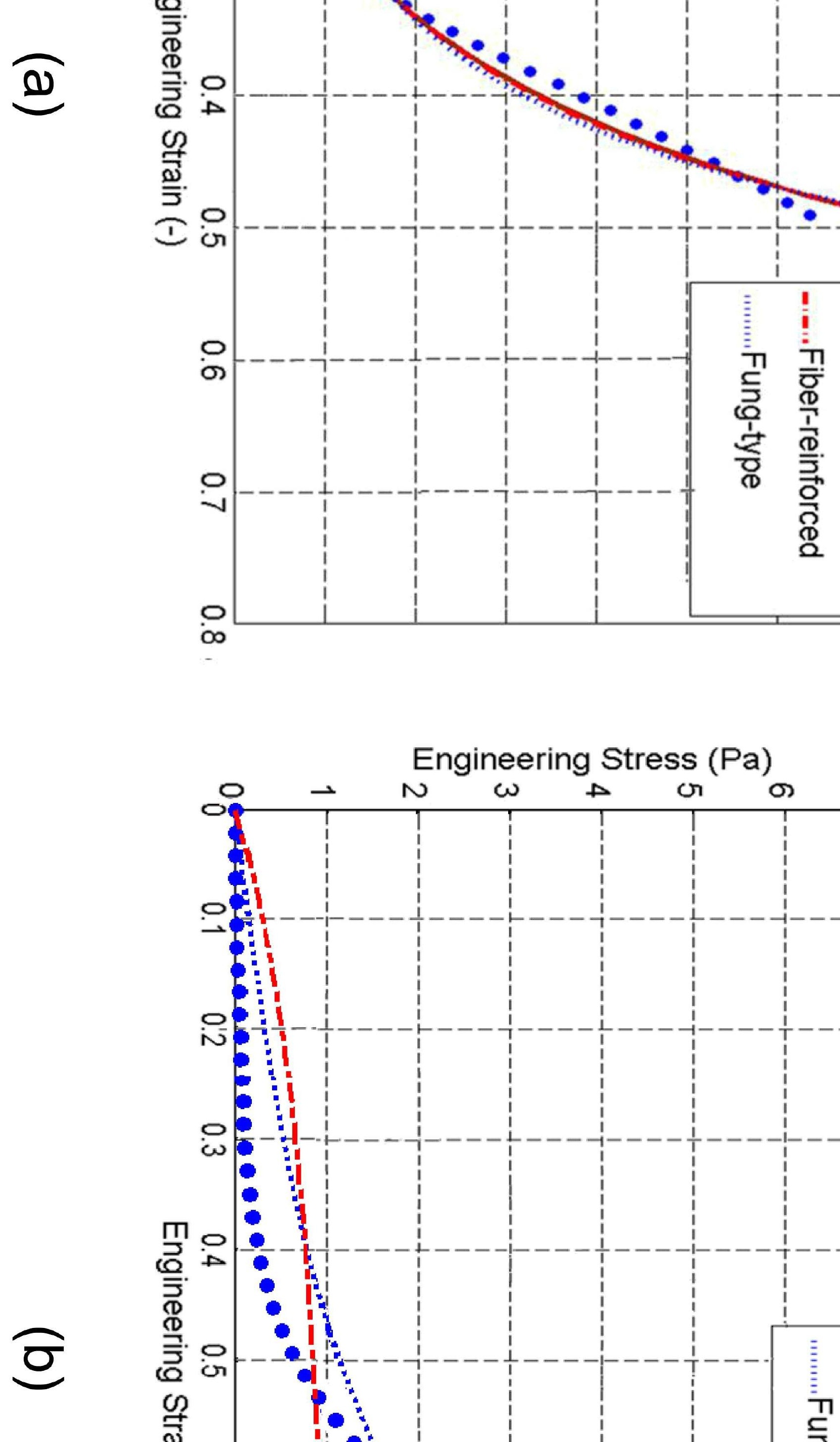


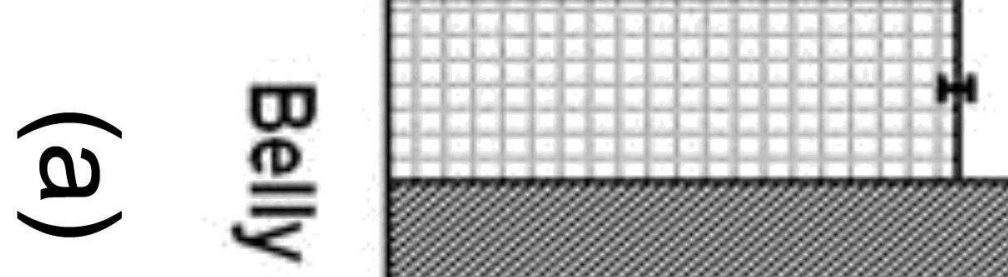

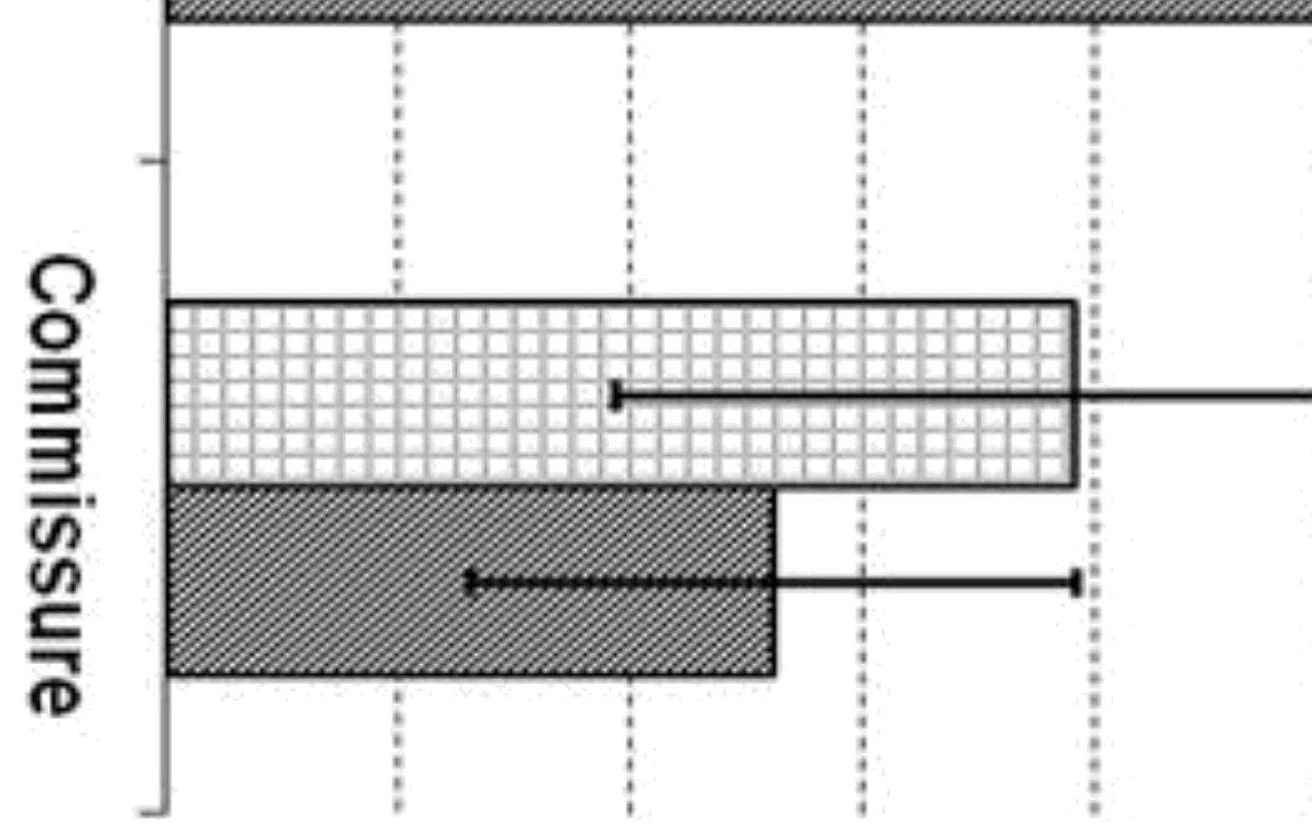

Effective Strain

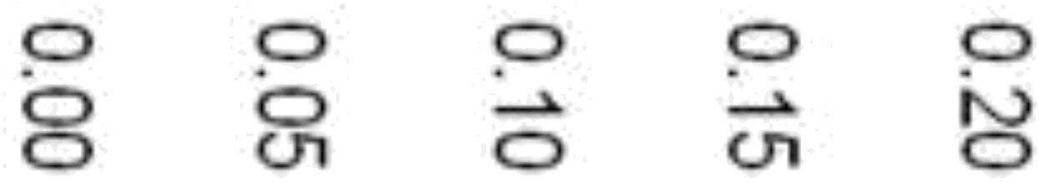

i

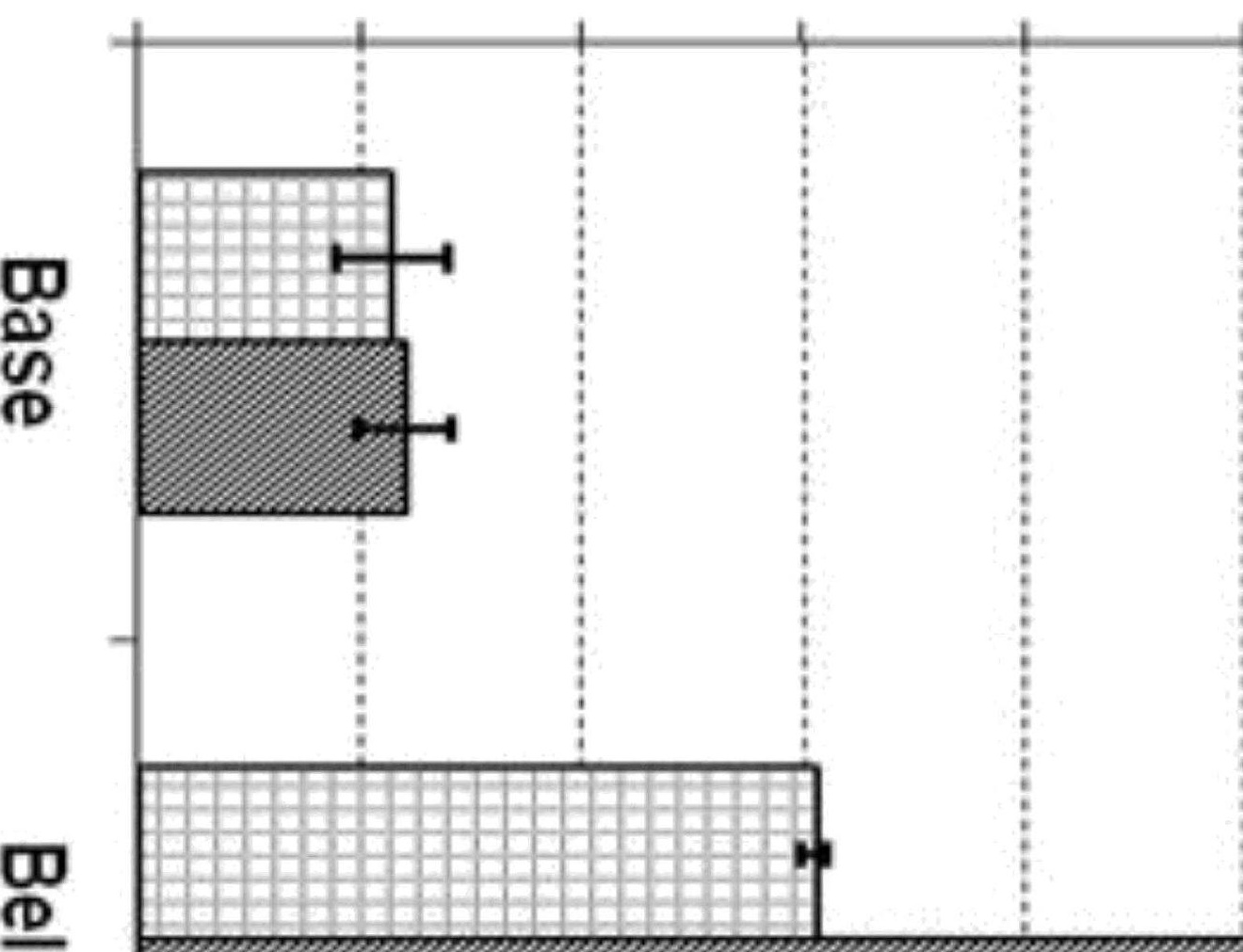



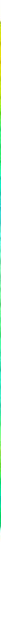

Maximum Principal Stress ( $\mathrm{Pa})$
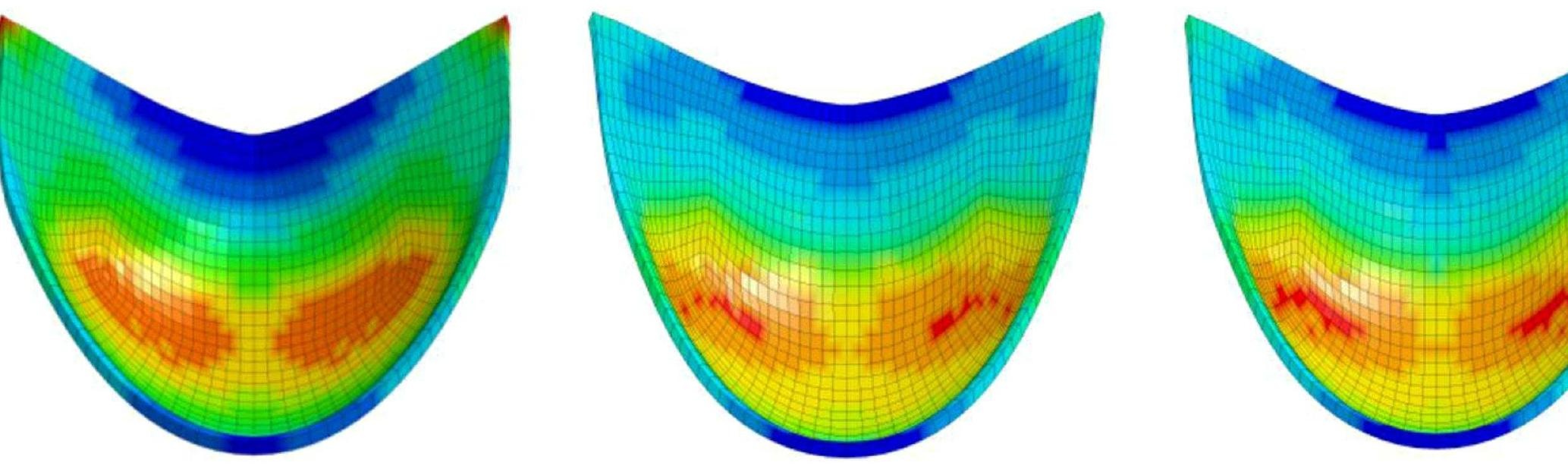

Effective Strain
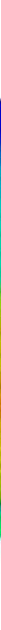

(a)

(b)

(c) 
Effective Strain
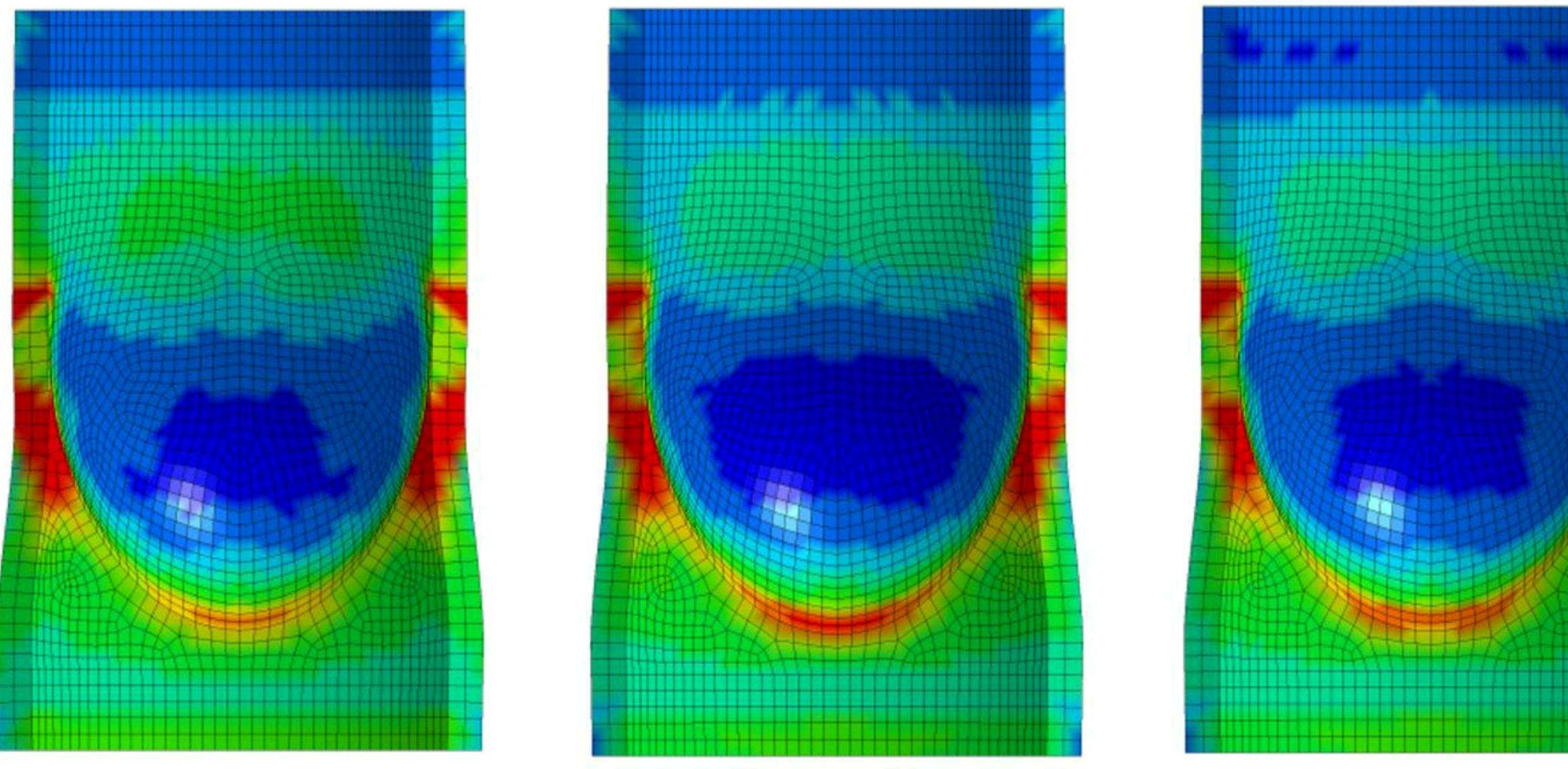

\section{Maximum Principal Stress $(\mathrm{Pa})$}
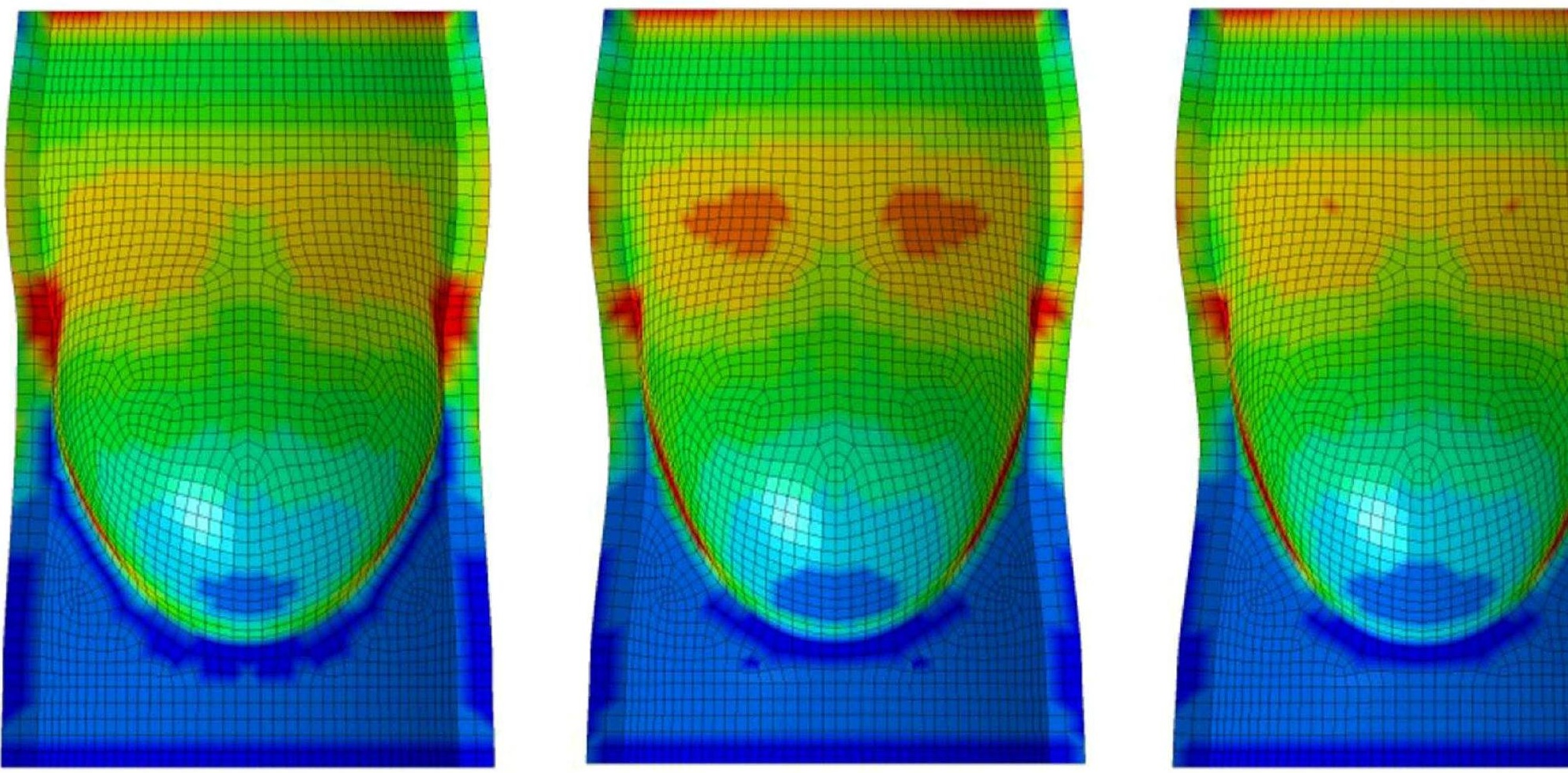

Effective Strain
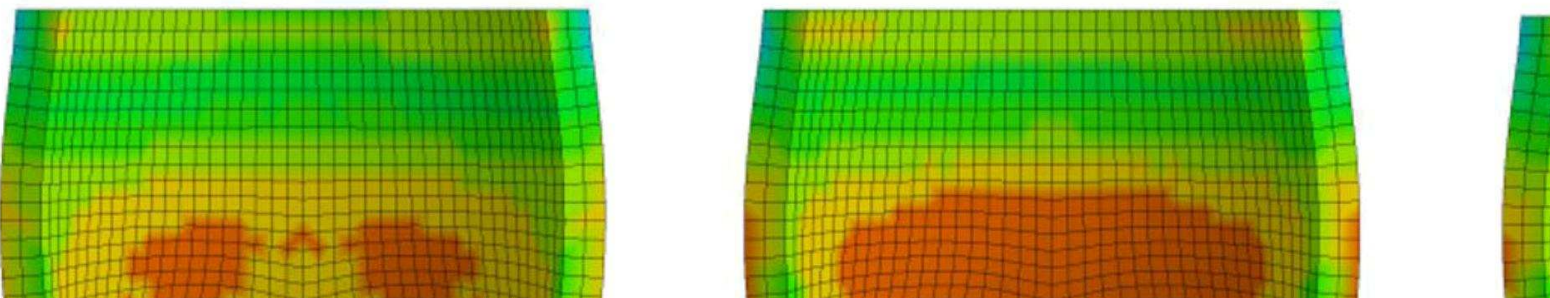


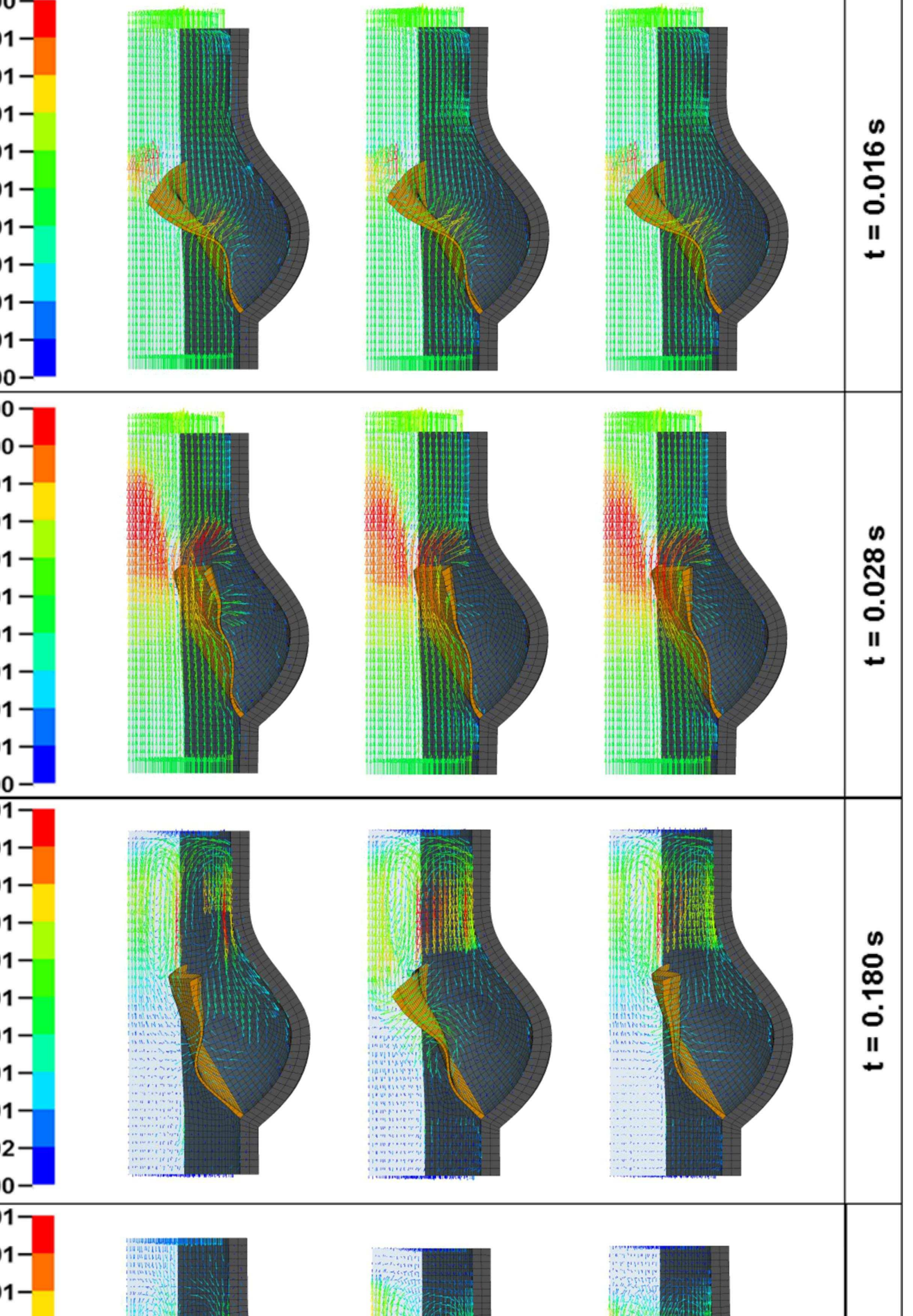


Tables

Table 1. Models used in the mesh sensitivity study.

\begin{tabular}{l|l|l|l}
\hline \hline model & Mod-1 & Mod-2 & Mod-3 \\
\hline Fluid elements & 8323 & 12332 & 97376 \\
\hline Solid elements & 2011 & 3063 & 5025 \\
\hline Leaflet layers & 1 & 1 & 2 \\
\hline \hline
\end{tabular}


Conflict of interest

The authors have no conflict of interest. 